\title{
Patterned Dynamics of Delay-Coupled Swarms with Random Communication Graphs
}

\author{
K. Szwaykowska, L. Mier-y-Teran-Romero and I. B. Schwartz
}

\begin{abstract}
Swarm and modular robotics are an emerging area in control of autonomous systems. However, coordinating a large group of interacting autonomous agents requires careful consideration of the logistical issues involved. In particular, inter-agent communication generally involves time delay, and bandwidth restrictions limit the number of neighbors with which each agent in the swarm can communicate. In this paper, we analyze coherent pattern dynamics of groups of delay-coupled agents, where the communication network is an Erdös-Renyi graph. We show that overall motion patterns for a globally-coupled swarm persist under decreasing network connectivity, and derive the bifurcation structure scaling relations for the emergence of different swarming behaviors as a function of the average network degree. We show excellent agreement between the theoretical scaling results and numerical simulations.
\end{abstract}

The emergence of complex dynamical behaviors from simple rules of pairwise interaction in aggregates of agents is a remarkable yet widespread phenomenon that appears in a multitude of application domains. In biology, aggregates form at all spatial and temporal scales, from the microscopic (ex., aggregates of bacterial cells or the collective motion of skin cells in wound healing) [1], [2], [3] to large-scale systems of fish, birds, and even humans [4], [5], [6]. In control systems, the emerging capabilities of swarms can allow groups of relatively small, inexpensive agents to achieve tasks that are beyond any individual agent. For example, aggregates of locally interacting agents have been proposed as a means to create scalable sensor arrays for surveillance and exploration [7], [8], [9], [10], [11], [12]; distributed sensing [13], [14]; cooperative construction [15], [16]; and the formation of reconfigurable modal systems [17], [18], [19].

The application of multi-agent systems for various tasks occurs in parallel with a modeling effort aimed at understanding the emergent properties of swarms. Existing literature on the subject provides a great selection of both individualbased [5], [6], [20], [4] and continuum models [21], [22], [2] of group motion. Many biologically-inspired models are based on "zones" of attraction, repulsion, and/or alignment interactions between agents [23], [24], [25]. Setting different ranges for attraction/repulsion interactions between all agents in the swarm or introducing heterogeneous dynamics can lead to interesting behaviors, such as splitting of a large swarm into smaller groups [26], [27], [28]. Swarming with nearest-neighbor alignment is studied numerically in [29].

US Naval Research Laboratory, Code 6792, Nonlinear System Dynamics Section, Plasma Physics Division, Washington, DC 20375. Email: klementyna.szwaykowska.ctr@nrl.navy.mil, lmieryt1@jhu.edu, ira.schwartz@nrl.navy.mil
Stochastic interactions between agents are modeled in [30].

Even so, most swarming models do not lend themselves to analysis, and there is still limited understanding of how group behaviors and structures arise out of simple interagent interactions. However, some progress has been made in understanding how the structure and parameters of a given swarm model contribute to the aggregate motion of the group. For example, [31] presents a simulation-based analysis of the different kinds of motion in a fish-schooling model; the authors map phase transitions between different aggregate behaviors as a function of group size and maximum number of neighbors that influence the motion of each "fish". In [6], the authors use simulations to study transitions in aggregate motions of prey in response to a predator attack.

In this paper, we are motivated by the idea of using a swarm of interacting autonomous aerial robots to conduct surveillance/monitoring of a specified environment, where a human operator uses a high-level control to guide the swarm as a whole (e.g., to set the monitoring region), while individual agent trajectories are governed by swarm interactions. The idea is similar to the reduced-order swarm control described in [32], [33] and references therein. Our goal is to rigorously characterize the swarm motion patterns under a simple but general swarming model, as a function of model parameters, so as to exploit them for parametric control of the system. We incorporate two key model modifications for real-world applicability: time delay and restricted communication bandwidth.

Systems of interacting individual agents, whether natural and engineered, involve some degree of communication delay [34], [35], [36], [37]. Time delay can have significant impact on system dynamics, leading to destabilization or synchronization of coupled systems [38], [39], [40]. As shown in our earlier work with globally delay-coupled swarms of homogeneous [41], [42], [43] and heterogeneous agents [44], communication delay can cause emergence of new collective motion patterns and, in the presence of noise, lead to switching between bistable patterns; this, in turn, can lead to instability in robotic swarming systems [25], [45].

Because global coupling is easier to analyze and a reasonable assumption in cases of high-bandwidth communication and when the number of agents is small, many models make the mathematically simple but physically implausible assumption that swarms are globally coupled (that is, each agent is influenced by the motion of all other agents in the swarm) [46], [47], [6], [48]. However, for large groups of agents, bandwidth restrictions generally mean that it is not feasible to maintain all-to-all communication. In this pa- 
per, we generalize our previous results for globally-coupled swarms by assuming an Erdös-Renyi communication network, where the mean number of neighbors for each agent in the swarm is fixed.

We use mean-field dynamics to analytically predict transitions between regimes of different collective swarm motions as a function of model parameters for swarms consisting of homogeneous delay-coupled agents with a fixed undirected Erdös-Renyi communication network. We use a simple yet general particle swarming model, which combines agent self-propulsion and inter-agent attraction. This choice can be justified in the case of swarming robots with very fast relaxation times, such as quadcopters, which can be treated as holonomic vehicles over the spatio-temporal scales required for a surveillance/monitoring deployment. We show that the stable motion patterns observed in the globallycoupled system (translation, ring formation, and rotation about a common center of mass) [42] persist under nonglobal coupling, for appropriately chosen model parameters. Our results are verified through numerical simulations.

\section{Problem Statement}

Consider a swarm of delay-coupled agents in the plane, with each agent indexed by $i$. We build an Erdös-Renyi communication network on the swarm as follows. Starting with a globally-coupled network, pick an existing link at random, using a uniform distribution over the existing links, and remove it, until the mean degree matches some target value. Let $\mathcal{N}_{i}$ denote the set of neighbors of agent $i$, that is, the set of agents with which agent $i$ shares a communication link. Because of communication/sensing delays, information about the position of the neighboring agents is available with delay $\tau$, assumed to be the same for all agents. For simplicity, we assume that all agents in the swarm have identical dynamics and that all connections in the network are bidirectional and fixed in time. The agents have selfpropulsion and are attracted to their neighbors with strength that depends on the coupling coefficient $a$. Then the motion of agent $i$ is governed by the following equation:

$$
\ddot{\mathbf{r}}_{i}=\left(1-\left|\dot{\mathbf{r}}_{i}\right|^{2}\right) \dot{\mathbf{r}}_{i}-\frac{a}{N} \sum_{j \in \mathcal{N}_{i}}\left(\mathbf{r}_{i}(t)-\mathbf{r}_{j}^{\tau}(t)\right),
$$

where superscript $\tau$ is used to denote time delay, so that $\mathbf{r}_{j}^{\tau}(t)=\mathbf{r}_{j}(t-\tau)$, and $|\cdot|$ denotes the Euclidean norm. The first term in the above equations represents the selfpropulsion of swarm agents. The second term models pairwise attraction between each agent and its neighbors in the swarm. This simplified model does not include short-range repulsion or other collision-avoidance strategies; however, earlier studies indicate that the collective dynamics of the swarm are not significantly altered by the introduction of short-range repulsion terms [42].

We examine the dynamics of the system analytically in the limit where the system is almost completely connected $\left(\frac{(N-1)-\operatorname{card}\left(\mathcal{N}_{i}\right)}{N-1} \ll 1\right.$, where $\operatorname{card}\left(\mathcal{N}_{i}\right)$ is the number of neighbors of node $i$ ), and show via simulations that the approximations made in the almost-connected limit hold closely even as the mean coupling degree is reduced to less than $50 \%$ of possible links.

\section{System Dynamics IN THE MEAN-FiEld}

In [42] we derived the mean-field dynamics of the system in the limit $N \rightarrow \infty$ where the delayed coupling was considered to be all-to-all. The difference considered in the model given by Eq. I is that each agent's motion depends only on its neighbors, rather than all other agents in the swarm. Let $\mathbf{R}(t)=\frac{1}{N} \sum_{i=1}^{N} \mathbf{r}_{i}(t)$ denote the position of the center of mass of the swarm, and let $\delta \mathbf{r}_{i}(t)=\mathbf{r}_{i}(t)-\mathbf{R}(t)$; note that $\sum_{i=1}^{N} \delta \mathbf{r}_{i}=0$. Let $\langle\cdot, \cdot\rangle$ denote the dot product in $\mathbb{R}^{2}$. The motion of the center of mass is governed by

$$
\begin{aligned}
\ddot{\mathbf{R}}= & \left(1-|\dot{\mathbf{R}}|^{2}\right) \dot{\mathbf{R}} \\
& -\frac{1}{N} \sum_{i=1}^{N}\left(|\dot{\mathbf{R}}|^{2} \delta \dot{\mathbf{r}}_{i}+\left(\left|\delta \dot{\mathbf{r}}_{i}\right|^{2}+2\left\langle\dot{\mathbf{R}}, \delta \dot{\mathbf{r}}_{i}\right\rangle\right)\left(\dot{\mathbf{R}}+\delta \dot{\mathbf{r}}_{i}\right)\right) \\
& -\frac{a \bar{p}}{N}\left(\mathbf{R}-\mathbf{R}^{\tau}\right)-\frac{a}{N^{2}} \sum_{i=1}^{N}\left(p_{i} \delta \mathbf{r}_{i}-\sum_{j \in \mathcal{N}_{i}} \delta \mathbf{r}_{j}^{\tau}\right),
\end{aligned}
$$

where $p_{i}=\operatorname{card}\left(\mathcal{N}_{i}\right)$ is the number of neighbors of agent $i$ (in the globally coupled case, $p_{i}=N-1$ ) and $\bar{p}=\frac{1}{N} \sum_{i=1}^{N} p_{i}$ is the mean degree of the network. We denote the mean fraction of missing links by $\varepsilon$, so that $\bar{p}=(1-\varepsilon)(N-1)$. Note that, if $p_{i}=p$ is the same for all $i$

$$
\sum_{i=1}^{N}\left(p \delta \mathbf{r}_{i}-\sum_{j \in \mathcal{N}_{i}} \delta \mathbf{r}_{j}^{\tau}\right)=-\sum_{i=1}^{N} \sum_{j \in \mathcal{N}_{i}} \delta \mathbf{r}_{j}^{\tau}=-p \sum_{i=1}^{N} \delta \mathbf{r}_{i}^{\tau}=0
$$

since $\sum_{i=1}^{N} \delta \mathbf{r}_{i}=0$ and in the double summation, each $\delta \mathbf{r}_{i}$ appears $p$ times because the network is undirected and each node has $p$ neighbors. However, in our network $p_{i}$ is drawn from a binomial distribution. Our previous work shows that in many instances either individual deviations from the center of mass are small, or in aggregate they average out over the whole population. (We discuss situations in which this assumption breaks in a later section). Then, neglecting all terms of order $\delta \mathbf{r}_{i}$ and in the limit $N \rightarrow \infty$, the center of mass motion is given approximately by

$$
\ddot{\mathbf{R}}=(1-|\dot{\mathbf{R}}|) \dot{\mathbf{R}}-a(1-\varepsilon)\left(\mathbf{R}(t)-\mathbf{R}^{\tau}(t)\right) .
$$

We note that the mean-field dynamics are identical to those for the globally-coupled homogeneous swarm described in [42], with coupling constant $a$ replaced by an 'effective' value $a_{\text {eff }}=a(1-\varepsilon)$. Quite remarkably, simulation results indicate that the system exhibits similar collective motions to the globally coupled case, even as $\bar{p}$ is significantly decreased. These basic collective motion patterns are "translation", where the entire swarm collapses to a point and travels along a straight-line trajectory at constant speed; "ring" motion, where the swarm agents form concentric counter-rotating rings about the stationary center of mass; and "rotating" motion, where the agents collapse to a small 
volume and collectively rotate about a fixed point. The collective motions of the swarm and the effects of nonglobal coupling are described in more detail in the following section.

\section{COLleCtive SWARM MOTIONS}

As in the globally coupled case, the steady-state motions of the swarm depend on values of the coupling coefficient $a$, the delay $\tau$; in addition, there is now a dependence on the fraction of missing links $\varepsilon$. The collective motion patterns of the swarm for different values of the parameters $a$ and $\tau$ are described in more detail below.

\section{A. Translating state}

In the translating state, the agent locations all lie close to the swarm center of mass, and the swarm moves with constant speed and direction. Following the calculation in [42], it can be shown that the translation speed $\|\dot{\mathbf{R}}\|$ must satisfy $\|\dot{\mathbf{R}}\|^{2}=1-a_{\text {eff }} \tau$. The system exhibits a pitchfork bifurcation at $a_{\text {eff }} \tau=1$, where the translating state disappears.

\section{B. Ring state}

For all values of $a$ and $\tau$, (4) admits a stationary solution, $\mathbf{R}(t)=\mathbf{R}(0)$. In this state, the agents converge to a pair of concentric, counter-rotating rings about the stationary center of mass. The mean radius and angular velocity of the agents in the ring state satisfy

$$
\begin{aligned}
& \rho=1 / \sqrt{a_{\mathrm{eff}}} \\
& \omega= \pm \sqrt{a_{\mathrm{eff}}},
\end{aligned}
$$

respectively (see Fig. 2). The stability of the ring state is determined by the characteristic equation

$$
M(\lambda ; a, \tau)=\left[\left(\lambda^{2}-\lambda+a_{\mathrm{eff}}\right)^{2}-\left(\lambda^{2}-\lambda+a_{\mathrm{eff}}\right) a_{\mathrm{eff}} e^{-\lambda \tau}\right]^{2}
$$

and is lost along Hopf bifurcation curves which give rise to a rotating state.

\section{Rotating state}

Solving for values where roots of $M$ cross the imaginary axis, we obtain Hopf bifurcation curves in the $a$ and $\tau$ plane:

$$
\tau=\frac{1}{\sqrt{2 a_{\mathrm{eff}}-1}}\left(\arctan \frac{\sqrt{2 a_{\mathrm{eff}}-1}}{1-a_{\mathrm{eff}}}+2 m \pi\right),
$$

$m \in \mathbb{Z}$. When $\varepsilon=0$, we recover the equations for the globally coupled system by taking $a_{\mathrm{eff}}=a$; the factor of $(1-\varepsilon)$ in $a_{\text {eff }}$ which results from breaking a fraction of the links in the global network represents a perturbation from the globally-coupled case (Fig. 11). Note that the pitchfork and Hopf bifurcation curves meet at a Bogdanov-Takens point when $a=\frac{1}{2(1-\varepsilon)}, \tau=2(1-\varepsilon)$.

The rotating state, in which the agents collapse and collectively rotate about a fixed point is created at the first Hopf bifurcation. In the case of global coupling, all agent positions coincide; however, when coupling is not global, different agents circle the fixed point with equal angular frequency but have different radii, and have a fixed relative phase offset from the center of mass, depending on their

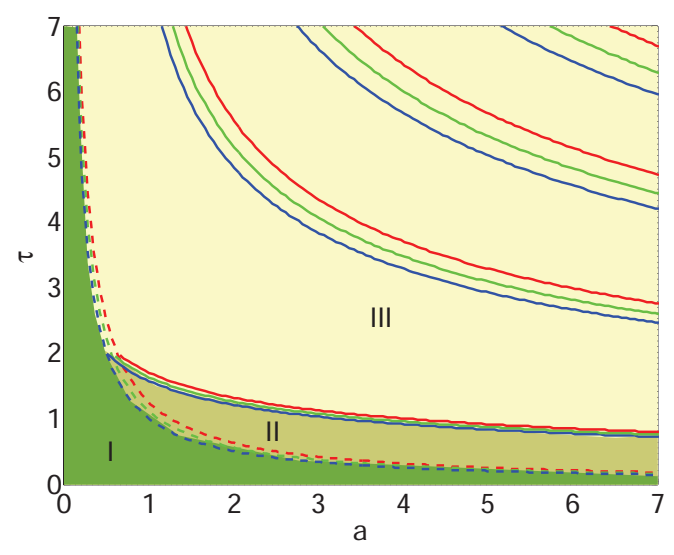

Fig. 1: Hopf bifurcation curves for $\varepsilon=0$ (blue), $\varepsilon=0.1$ (green), and $\varepsilon=0.2$ (red). The dashed lines show the location of the pitchfork bifurcation where the translating state disappears. In the mean field, the translating state occurs in region I; the ring state in region II, and the rotating state, in region III.

coupling degree (see Fig. 3). The radius and angular velocity of the center of mass of the swarm in the rotating state satisfy

$$
\begin{aligned}
\rho & =\frac{1}{|\omega|} \sqrt{1-a_{\mathrm{eff}} \frac{\sin \omega \tau}{\omega}} \\
\omega^{2} & =a_{\mathrm{eff}}(1-\cos \omega \tau)
\end{aligned}
$$

(see Fig. 4).

We now investigate the precise spatial organization of swarming agents in the rotating state. Using $\mathbf{r}_{j}^{\tau}=\mathbf{R}^{\tau}+\delta \mathbf{r}_{j}^{\tau}$ allows us to write the coupling of agent $i$ to the other particles as

$$
\begin{aligned}
\sum_{j \in \mathcal{N}_{i}}\left(\mathbf{r}_{i}(t)-\mathbf{r}_{j}^{\tau}(t)\right) & =p_{i}\left(\mathbf{r}_{i}-\mathbf{R}^{\tau}\right)-\sum_{j \in \mathcal{N}_{i}} \delta \mathbf{r}_{j}^{\tau} \\
& \approx p_{i}\left(\mathbf{r}_{i}-\mathbf{R}^{\tau}\right),
\end{aligned}
$$

after neglecting the sum of small terms $\delta \mathbf{r}_{j}^{\tau}$. In this way, the dynamics of agent $i$ becomes

$$
\ddot{\mathbf{r}}_{i}=\left(1-\left|\dot{\mathbf{r}}_{i}\right|^{2}\right) \dot{\mathbf{r}}_{i}-\frac{a p_{i}}{N}\left(\mathbf{r}_{i}(t)-\mathbf{R}^{\tau}(t)\right),
$$

so that dynamically, less-than-global coupling in our ErdösRenyi network is approximately equivalent to a fully connected network of agents with heterogeneous coupling coefficients

$$
a_{i}=a p_{i} /(N-1),
$$

where the factor of $N-1$ is introduced so that $a_{i}=a$ in the fully connected case where $p_{i}=N-1$. In the limit $N \rightarrow \infty$, we have

$$
\ddot{\mathbf{r}}_{i}=\left(1-\left|\dot{\mathbf{r}}_{i}\right|^{2}\right) \dot{\mathbf{r}}_{i}-a_{i}\left(\mathbf{r}_{i}-\mathbf{R}^{\tau}\right),
$$

where the motion of the center of mass $\mathbf{R}$ is given by (4).

Let $(\rho, \theta)$ and $\left(\rho_{i}, \theta_{i}\right)$ denote the polar coordinates of the swarm center of mass and of agent $i$, respectively. In these 

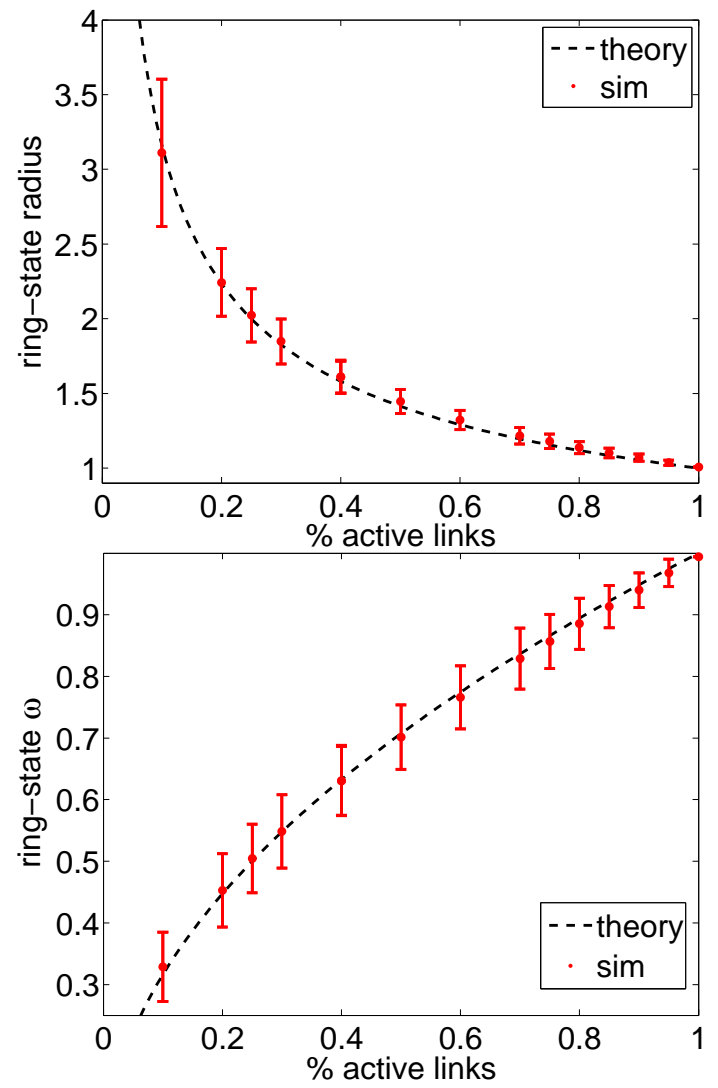

Fig. 2: Comparison of the theoretical values of radius (top) and angular velocity (bottom) of the agents in the ring state, for different connection degrees, compared with fullswarm simulation of 150 agents. The $\mathrm{x}$-axis represents the percentage of active links, out of all possible links; all links are bidirectional and time-invariant. Error bars are shown one standard deviation above and below the mean values. The simulations were run with $a=1$ and $\tau=2.5$.

coordinates, the equations of motion for the swarm are

$$
\begin{aligned}
\ddot{\rho} & =\left(1-\rho^{2} \dot{\theta}^{2}-\dot{\rho}^{2}\right) \dot{\rho}+\rho \dot{\theta}^{2}-\bar{a}\left(\rho-\rho^{\tau} \cos \left(\theta-\theta^{\tau}\right)\right) \\
\ddot{\theta} & =\left(1-\rho^{2} \dot{\theta}^{2}-\dot{\rho}^{2}\right) \rho \dot{\theta}-2 \dot{\rho} \dot{\theta}-\bar{a} \rho^{\tau} \sin \left(\theta-\theta^{\tau}\right) \\
\ddot{\rho}_{i} & =\left(1-\rho_{i}^{2} \dot{\theta}_{i}^{2}-\dot{\rho}_{i}^{2}\right) \dot{\rho}_{i}+\rho_{i} \dot{\theta}_{i}^{2}-a_{i}\left(\rho_{i}-\rho^{\tau} \cos \left(\theta_{i}-\theta^{\tau}\right)\right) \\
\rho_{i} \ddot{\theta}_{i} & =\left(1-\rho_{i}^{2} \dot{\theta}_{i}^{2}-\dot{\rho}_{i}^{2}\right) \rho_{i} \dot{\theta}_{i}-2 \dot{\rho}_{i} \dot{\theta}_{i}-a_{i} \rho^{\tau} \sin \left(\theta_{i}-\theta^{\tau}\right),
\end{aligned}
$$

where $\bar{a}=\frac{1}{N} \sum_{i=1}^{N} a_{i}$ is the mean coupling coefficient.

Without loss of generality, we set the origin of the polar coordinate system at the center of rotation so that $\rho$ and $\rho_{i}$ are constant in time. Also, we have $\ddot{\theta}=\ddot{\theta}_{i} \equiv 0$. Let $\omega=\dot{\theta}$ and $\omega_{i}=\dot{\theta}_{i}$. For convenience, we define $\xi_{a}^{i}=a_{i} / \bar{a}$ and

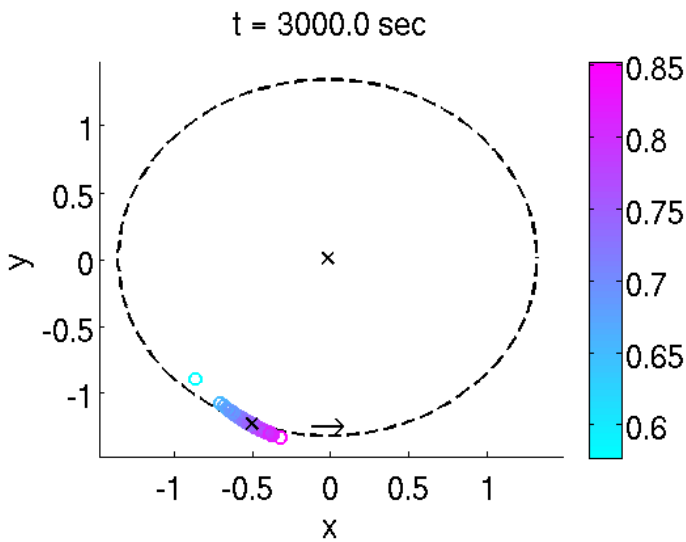

Fig. 3: Snapshot of simulation showing swarm in rotating state about a stationary center point (marked by ' $x$ '). The colors indicate the coupling degree of each agent, normalized by $N-1$. Here $a=1, \tau=4.5$, and $\bar{p} /(N-1)=0.75$.

$\xi_{\rho}^{i}=\rho_{i} / \rho$. The equations of motion then become

$$
\begin{aligned}
0= & \rho \omega^{2}-\bar{a} \rho(1-\cos (\omega \tau)) \\
0= & \left(1-\rho^{2} \omega^{2}\right) \rho \omega-\bar{a} \rho \sin (\omega \tau) \\
0= & \xi_{\rho}^{i} \rho \omega_{i}^{2}-\xi_{a}^{i} \bar{a}\left(\xi_{\rho}^{i} \rho\right. \\
& \left.\quad-\rho \cos \left(\left(\omega_{i}-\omega\right) t+\theta_{i}(0)-\theta(0)+\omega \tau\right)\right) \\
0= & \left(1-\left(\xi_{\rho}^{i}\right)^{2} \rho^{2} \omega_{i}^{2}\right) \xi_{\rho}^{i} \rho \omega_{i} \\
& \left.\quad-\xi_{a}^{i} \bar{a} \rho \sin \left(\left(\omega_{i}-\omega\right) t+\theta_{i}(0)-\theta(0)+\omega \tau\right)\right) .
\end{aligned}
$$

Note that equations 21 and 22 can only be satisfied for all times $t$ if $\omega_{i}=\omega$. Let $\Delta \theta_{i}=\theta_{i}(0)-\theta(0)$ denote the angular offset between particle $i$ and the center of mass. Simplifying, we finally have

$$
\begin{aligned}
& 0=\omega^{2}-\bar{a}(1-\cos (\omega \tau)) \\
& 0=\left(1-\rho^{2} \omega^{2}\right) \omega-\bar{a} \sin (\omega \tau) \\
& 0=\xi_{\rho}^{i} \omega^{2}-\xi_{a}^{i} \bar{a}\left(\xi_{\rho}^{i}-\cos \left(\omega \tau+\Delta \theta_{i}\right)\right) \\
& \left.0=\left(1-\left(\xi_{\rho}^{i}\right)^{2} \rho^{2} \omega^{2}\right) \xi_{\rho}^{i} \omega-\xi_{a}^{i} \bar{a} \sin \left(\omega \tau+\Delta \theta_{i}\right)\right) .
\end{aligned}
$$

This set of coupled nonlinear equations can be solved numerically for $\rho, \omega, \xi_{\rho}^{i}$, and $\Delta \theta_{i}$ for different values of $\bar{a}, \tau$ and $\xi_{a}^{i}$. We compare numerical solutions for the swarm center of mass radius and angular velocity with simulations of a swarm with Erdös-Renyi communication network structure (see Fig. 4). Solution curves for $\xi_{\rho}^{i}$ and $\Delta \theta_{i}$ for different values of $\overline{\bar{a}}$ are shown in Fig. 5, a comparison with simulation results is shown in Fig. 6. The slight discrepancy in the rotating state radius in Fig. 4 and in $\xi_{\rho}^{i}$ in Fig. 6 is understood as follows. The radius of the center of mass computed from (23) and (24) assumes that agent positions' deviate only slightly from the center of mass. However, as the mean coupling coefficient decreases the agents become spread out over an extended arc (as seen in Fig. 3) and our assumption becomes invalid. In this 'arc' configuration the center of mass of the swarm moves closer towards the center of rotation than theory predicts. The analogue to a system with perturbed coupling coefficient breaks down here; 
for a globally-connected swarm with decreasing coupling coefficient $a$, the rotating state disappears when the system crosses the curve $\bar{a} \tau^{2}=2$, where the rotating state radius diverges. The swarm then transitions to a translating state. It is, however, remarkable, that the mean-field analysis captures so much of the overall swarm behavior even as the coupling degree is significantly decreased.
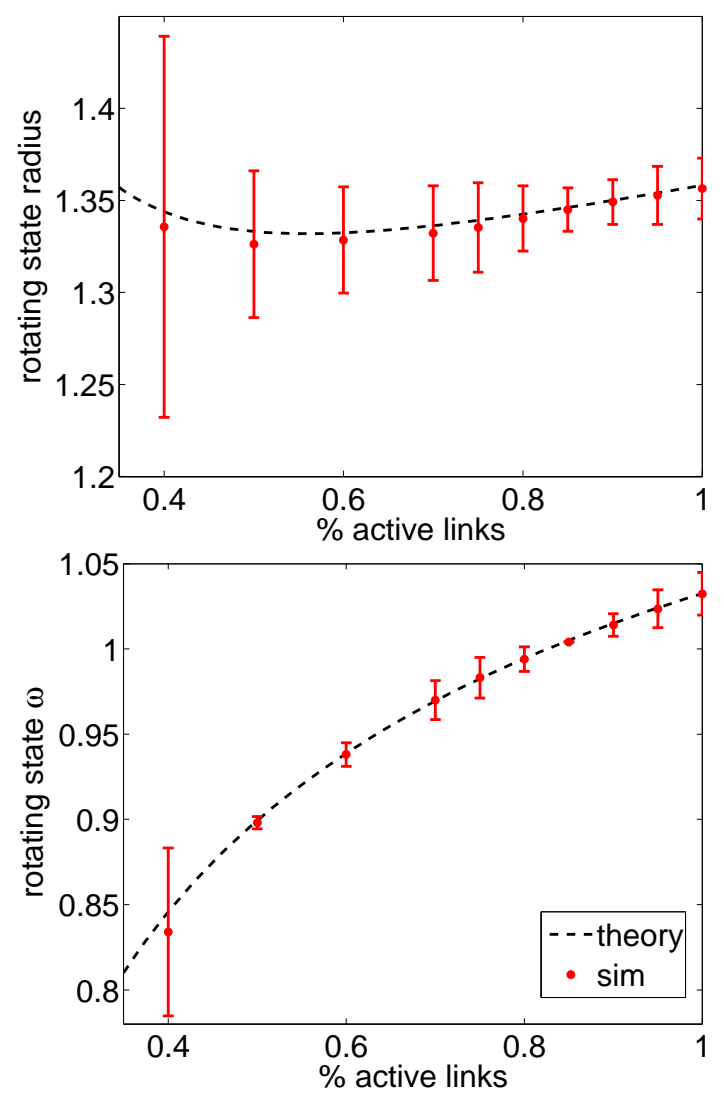

Fig. 4: Comparison of the theoretical values of radius (top) and angular velocity (bottom) of the agents in the rotating state with full-swarm simulations of 150 agents, for different connection degrees. The simulation values are obtained by averaging over all agents int he swarm. The $\mathrm{x}$-axis represents the percentage of active links, out of all possible links; all links are bidirectional. Error bars are shown one standard deviation above and below the mean.

\section{CONCLUSION}

In this paper we have analyzed the collective motion patterns of a swarm with Erdös-Renyi communication network structure, using a mean-field approach from statistical physics, with the assumption that the number of agents goes to infinity. We derived bifurcation diagrams demarcating regions of different collective motions, for different values of mean degree in the communication network. We showed that behaviors described in [42] for the globally-coupled swarm, namely translation, ring state, and rotation, persist as communication links are broken, even though the bifurcation curves are shifted as coupling degree of the network decreases.

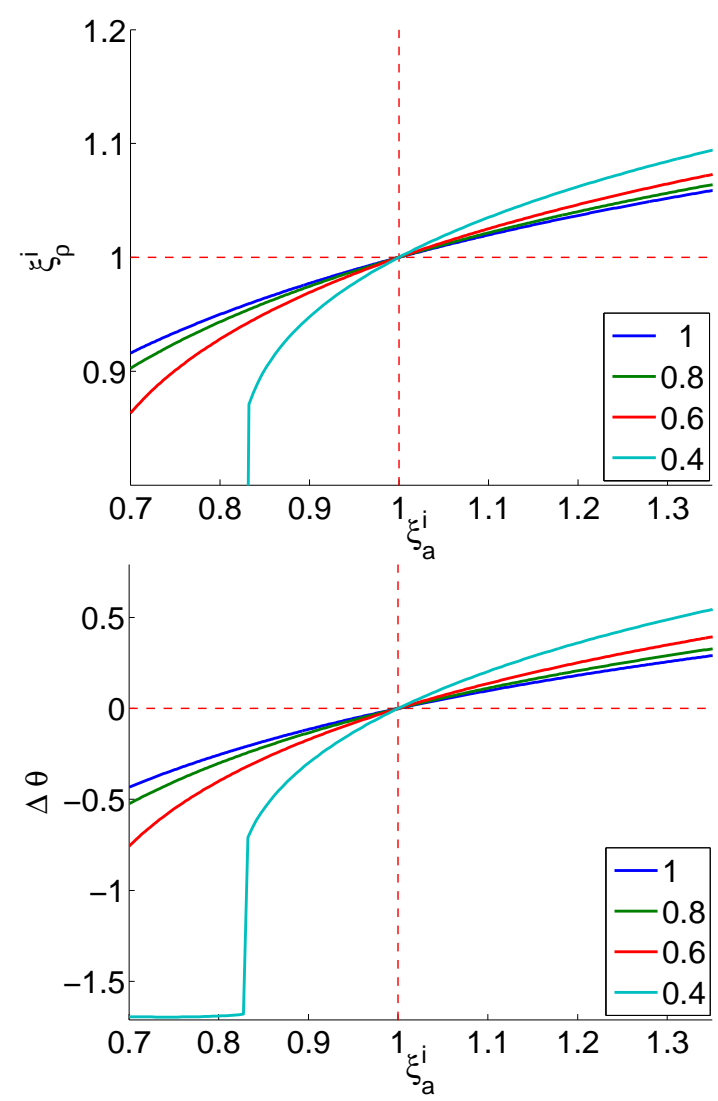

Fig. 5: Theoretical values for ratio of radius for agent $i$ to radius of swarm center of mass (top) and phase difference (bottom) as a function of $\xi_{a}^{i}=a_{i} / \bar{a}$, for $\bar{a}=1,0.8,0.6$, and 0.4 .

We derive expressions for the speed of the swarm in the translating state as a function of time delay and coupling coefficient; for the mean radius and angular velocity of agents in the ring state; and for the angular velocity, and individual radii and phase offsets for individual agents in the rotating state. We have verified these calculations with simulations of the full-swarm dynamics. It is remarkable that our model reduction, which starts with $N$ second-order delay-differential equations and yields one equation of the same type, is able to quantitatively capture so many aspects of the full swarm dynamics, even as the coupling degree of agents within the swarm is significantly decreased.

In the case that many agents are coordinating together, limited communication bandwidth makes all-to-all communication infeasible, and may lead to significant communication delays. By dropping the requirement for all-to-all communication used in our previous work, the current paper brings us one step closer to a possible implementation of swarming control algorithms for very large aggregates of agents. Understanding the natural emerging dynamics of the system in these circumstances allows us to exploit them when designing controls for swarming applications.

In future work, we will study the effects of using nearestneighbor communication rather than the random Erdös-Renyi 

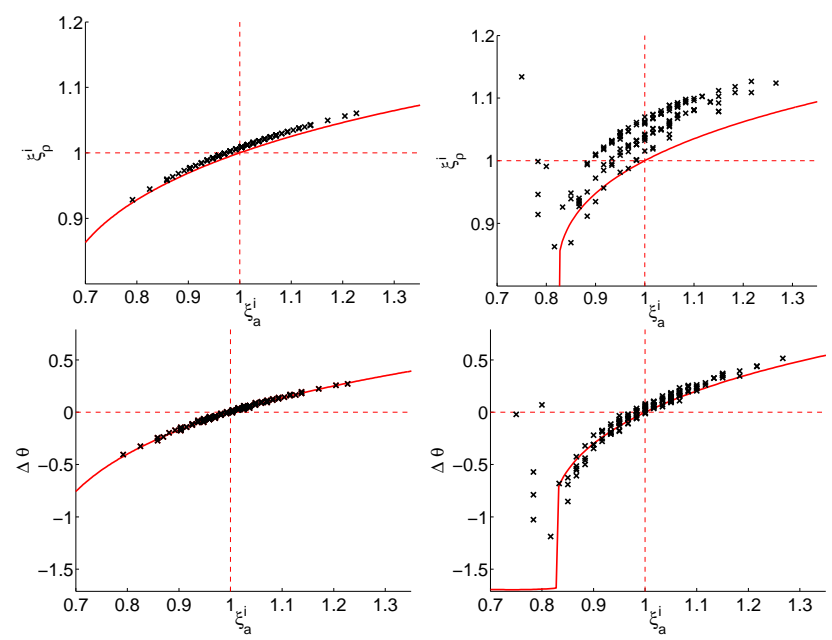

Fig. 6: Comparison of simulation and theoretical values for radius (top) and phase difference from the center of mass (bottom) for agent $i$. Results are shown for two swarms, the first with $\bar{a}=0.6$ (left) and the second with $\bar{a}=0.4$ (right) (that is, $60 \%$ of links active and $40 \%$ of links active, respectively). For both swarms, $\tau=4.5 \mathrm{sec}$, and number of agents is 150. All links are bidirectional. Note that within each swarm, agents with higher coupling degree lie further from the center of rotation.

communication network used here. We will verify our results in the lab using a swarm of quadcopters operating in a mixed real-virtual environment, in which arbitrarily large numbers of virtual agents can be simulated to interact with the group of real vehicles. We will test how our results scale with the number of agents in the network, and apply parametric control for dynamic pattern-switching.

\section{ACKNOWLEDGMENTS}

This research was performed while KS held a National Research Council Research Associateship Award at the U.S. Naval Research Laboratory. This research is funded by the Office of Naval Research contract no. N0001412WX2003 and the Naval Research Laboratory 6.1 program contract no. N0001412WX30002. LMR is a post-doctoral fellow at Johns Hopkins University supported by the National Institutes of Health.

\section{REFERENCES}

[1] E. O. Budrene and H. C. Berg, "Dynamics of formation of symmetrical patterns by chemotactic bacteria," Nature, vol. 376, pp. 49-53, 1995.

[2] A. A. Polezhaev, R. A. Pashkov, A. I. Lobanov, and I. B. Petrov, "Spatial patterns formed by chemotactic bacteria Escherichia coli," The International Journal of Developmental Biology, vol. 50, pp. 309-314, Jan. 2006.

[3] R. M. Lee, D. H. Kelley, K. N. Nordstrom, N. T. Ouellette, and W. Losert, "Quantifying stretching and rearrangement in epithelial sheet migration.," New Journal of Physics, vol. 15, Feb. 2013.

[4] K. r. Tunstrø m, Y. Katz, C. C. Ioannou, C. Huepe, M. J. Lutz, and I. D. Couzin, "Collective states, multistability and transitional behavior in schooling fish," PLoS computational biology, vol. 9, p. e1002915, Jan. 2013.

[5] D. Helbing and P. Molnar, "Social force model for pedestrian dynamics," Physical Review E, vol. 51, no. 5, pp. 4282-4286, 1995.
[6] S.-H. Lee, "Predator's attack-induced phase-like transition in prey flock," Physics Letters A, vol. 357, pp. 270-274, Sept. 2006.

[7] P. Bhatta, E. Fiorelli, F. Lekien, N. E. Leonard, D. A. Paley, F. Zhang, R. Bachmayer, D. M. Fratantoni, R. E. Davis, and R. J. Sepulchre, "Coordination of an underwater glider fleet for adaptive sampling," in Proceedings of the International Workshop on Underwater Robotics, no. August, pp. 61-69, 2005.

[8] Y.-1. Chuang, M. R. DOrsogna, D. Marthaler, A. L. Bertozzi, and L. S. Chayes, "State transitions and the continuum limit for a 2D interacting, self-propelled particle system," Physica D: Nonlinear Phenomena, vol. 232, pp. 33-47, Aug. 2007.

[9] K. M. Lynch, I. B. Schwartz, P. Yang, and R. A. Freeman, "Decentralized Environmental Modeling by Mobile Sensor Networks," IEEE Transactions on Robotics, vol. 24, pp. 710-724, June 2008.

[10] P. Yang, R. A. Freeman, and K. M. Lynch, "Multi-agent coordination by decentralized estimation and control," IEEE Transactions on Automatic Control, vol. 53, no. 11, pp. 2480-2496, 2008.

[11] W. Wu and F. Zhang, "Cooperative exploration of level surfaces of three dimensional scalar fields," Automatica, vol. 47, pp. 2044-2051, Sept. 2011.

[12] W. Wu and F. Zhang, "Robust Cooperative Exploration With a Switching Strategy," IEEE Transactions on Robotics, vol. 28, no. 4, pp. 828-839, 2012.

[13] T. H. Chung, J. W. Burdick, and R. M. Murray, "A decentralized motion coordination strategy for dynamic target tracking," in IEEE International Conference on Robotics and Automation, vol. 2006, pp. 2416-2422, 2006.

[14] S. Kar and J. M. F. Moura, "Distributed linear parameter estimation in sensor networks: Convergence properties," in 2008 42nd Asilomar Conference on Signals, Systems and Computers, pp. 1347-1351, Ieee, Oct. 2008.

[15] F. Augugliaro, S. Lupashin, M. Hamer, C. Male, M. Hehn, M. W. Mueller, J. S. Willmann, F. Gramazio, M. Kohler, and R. D’Andrea, "The flight assembled architecture installation: Cooperative contruction with flying machines," IEEE Control Systems, vol. 34, no. August, pp. 46-64, 2014.

[16] J. Werfel, K. Petersen, and R. Nagpal, "Designing collective behavior in a termite-inspired robot construction team," Science (New York, N.Y.), vol. 343, pp. 754-8, Feb. 2014.

[17] S. Bandyopadhyay, S.-j. Chung, and F. Y. Hadaegh, "Probabilistic Swarm Guidance using Inhomogeneous Markov Chains," 2014.

[18] M. Dorigo, D. Floreano, L. M. Gambardella, F. Mondada, S. Nolfi, T. Baaboura, M. Birattari, M. Bonani, M. Brambilla, A. Brutschy, D. Burnier, A. Campo, A. L. Christensen, A. Decugniere, G. Di Caro, F. Ducatelle, E. Ferrante, A. Forster, J. M. Gonzales, J. Guzzi, V. Longchamp, S. Magnenat, N. Mathews, M. Montes de Oca, R. O’Grady, C. Pinciroli, G. Pini, P. Retornaz, J. Roberts, V. Sperati, T. Stirling, A. Stranieri, T. Stutzle, V. Trianni, E. Tuci, A. E. Turgut, and F. Vaussard, "Swarmanoid: A Novel Concept for the Study of Heterogeneous Robotic Swarms," IEEE Robotics \& Automation Magazine, vol. 20, pp. 60-71, Dec. 2013.

[19] M. Rubenstein, A. Cornejo, and R. Nagpal, "Programmable selfassembly in a thousand-robot swarm," Science, vol. 345, pp. 795-799, Aug. 2014.

[20] T. Vicsek, A. Czirok, E. Ben-Jacob, I. Cohen, and O. Shochet, "Novel type of phase transition in a system of self-driven particles," 2006.

[21] L. Edelstein-Keshet, D. Grunbaum, and J. Watmough, "Do travelling band solutions describe cohesive swarms? An investigation for migratory locusts," Journal of Mathematical Biology, vol. 36, pp. 515-549, July 1998.

[22] C. M. Topaz and A. L. Bertozzi, "Swarming Patterns in a TwoDimensional Kinematic Model for Biological Groups," SIAM Journal on Applied Mathematics, vol. 65, pp. 152-174, Jan. 2004.

[23] J. M. Miller, A. Kolpas, J. P. J. Neto, and L. F. Rossi, "A continuum three-zone model for swarms," Bulletin of mathematical biology, vol. 74, pp. 536-61, Mar. 2012.

[24] I. Tarras, N. Moussa, M. Mazroui, Y. Boughaleb, and A. Hajjaji, "Collective Behavior of Interacting Particles: Radius-Dependent Phase Transition,” Modern Physics Letters B, vol. 27, p. 1350028, Feb. 2013.

[25] C. Virágh, G. Vásárhelyi, N. Tarcai, T. Szörényi, G. Somorjai, T. Nepusz, and T. Vicsek, "Flocking algorithm for autonomous flying robots," Bioinspiration \& biomimetics, vol. 9, p. 025012, June 2014.

[26] M. Kumar, D. P. Garg, and V. Kumar, "Segregation of Heterogeneous Units in a Swarm of Robotic Agents," IEEE Transactions on Automatic Control, vol. 55, no. 3, pp. 743-748, 2010. 
[27] Z. Chen, T. Chu, and J. Zhang, "Swarm splitting and multiple targets seeking in multi-agent dynamic systems," 49th IEEE Conference on Decision and Control (CDC), pp. 4577-4582, Dec. 2010.

[28] Z. Chen, H. Liao, and T. Chu, "Clustering in multi-agent swarms via medium-range interaction,” EPL (Europhysics Letters), vol. 96, p. 40015, Nov. 2011.

[29] T. Vicsek, A. Czirok, E. Ben-Jacob, I. Cohen, and O. Shochet, "Novel Type of Phase Transition in a System of Self-Driven Particles," Physical Review Letters, vol. 75, no. 6, pp. 1226-1229, 1995.

[30] C. Nilsen, J. Paige, O. Warner, B. Mayhew, R. Sutley, M. Lam, A. J. Bernoff, and C. M. Topaz, "Social aggregation in pea aphids: experiment and random walk modeling," PloS ONE, vol. 8, p. e83343, Jan. 2013.

[31] S. V. Viscido, J. K. Parrish, and D. Grünbaum, "The effect of population size and number of influential neighbors on the emergent properties of fish schools," Ecological Modelling, vol. 183, pp. 347363, Apr. 2005.

[32] C. Belta and V. Kumar, "Abstraction and control for groups of robots," IEEE Transactions on Robotics, vol. 20, no. 5, pp. 865-875, 2004.

[33] N. Michael, C. Belta, and V. Kumar, "Controlling three dimensional swarms of robots," Proceedings - IEEE International Conference on Robotics and Automation, vol. 2006, no. May, pp. 964-969, 2006.

[34] A. Martin and S. Ruan, "Predator-prey models with delay and prey harvesting," Journal of Mathematical Biology, vol. 43, pp. 247-267, Sept. 2001.

[35] S. Bernard, J. Bélair, and M. C. Mackey, "Bifurcations in a whiteblood-cell production model," Comptes Rendus Biologies, vol. 327, pp. 201-210, Mar. 2004.

[36] N. A. M. Monk, "Oscillatory Expression of Hes1, p53, and NF-kB Driven by Transcriptional Time Delays," Current Biology, vol. 13, pp. 1409-1413, 2003.

[37] E. Forgoston and I. B. Schwartz, "Delay-induced instabilities in selfpropelling swarms," Physical Review E, vol. 77, no. 302, p. 035203 , 2008.

[38] A. Papachristodoulou and A. Jadbabaie, "Synchronization in Oscillator Networks with Heterogeneous Delays, Switching Topologies and Nonlinear Dynamics," in Proceedings of the 45th IEEE Conference on Decision and Control, pp. 4307-4312, Ieee, 2006.

[39] Z. Zuo, C. Yang, and Y. Wang, "A unified framework of exponential synchronization for complex networks with time-varying delays," Physics Letters A, vol. 374, no. 19-20, pp. 1989-1999, 2010.

[40] A. Englert, S. Heiligenthal, W. Kinzel, and I. Kanter, "Synchronization of chaotic networks with time-delayed couplings: An analytic study," Physical Review E - Statistical, Nonlinear, and Soft Matter Physics, vol. 83, no. 4, pp. 1-14, 2011.

[41] L. Mier-y-Teran Romero, E. Forgoston, and I. B. Schwartz, "Noise, Bifurcations, and Modeling of Interacting Particle Systems," in Proceedings of the IEEE/RSJ International Conference on Intelligent Robots and Systems, pp. 3905-3910, Jan. 2011.

[42] L. Mier-y-Teran Romero, E. Forgoston, and I. B. Schwartz, "Coherent Pattern Prediction in Swarms of Delay-Coupled Agents," IEEE Transactions on Robotics, vol. 28, pp. 1034-1044, Oct. 2012.

[43] B. S. Lindley, L. Mier-y-Teran Romero, and I. B. Schwartz, "Noise Induced Pattern Switching in Randomly Distributed Delayed Swarms," in Proc. 2013 American Control Conference (ACC 2013), pp. 45874591, 2013.

[44] K. Szwaykowska, L. Mier-y-Teran Romero, and I. B. Schwartz, "Collective motions of heterogeneous swarms," IEEE Transactions on Automation Science and Engineering (to appear), Sept. 2015.

[45] Y. Liu, K. M. Passino, and M. Polycarpou, "Stability analysis of onedimensional asynchronous swarms," IEEE Transactions on Automatic Control, vol. 48, no. 10, pp. 1848-1854, 2003.

[46] S. Motsch and E. Tadmor, "A New Model for Self-organized Dynamics and Its Flocking Behavior," Journal of Statistical Physics, vol. 144, pp. 923-947, Aug. 2011.

[47] Y. Chen and T. Kolokolnikov, "A minimal model of predator-swarm interactions.," Journal of the Royal Society, Interfacelthe Royal Society, vol. 11, p. 20131208, May 2014.

[48] F. Vecil, P. Lafitte, and J. Rosado Linares, "A numerical study of attraction/repulsion collective behavior models: 3D particle analyses and 1D kinetic simulations," Physica D, vol. 260, pp. 127-144, 2013. 